\title{
Electron transport in a starburst oxadiazole
}

Cite as: Journal of Applied Physics 82, 4957 (1997); https://doi.org/10.1063/1.366396 Submitted: 17 April 1997 . Accepted: 06 August 1997 . Published Online: 04 June 1998

J. Bettenhausen, P. Strohriegl, W. Brütting, H. Tokuhisa, and T. Tsutsui

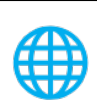

\section{ARTICLES YOU MAY BE INTERESTED IN}

Efficient, deep-blue organic electrophosphorescence by guest charge trapping Applied Physics Letters 83, 3818 (2003); https://doi.org/10.1063/1.1624639

High-efficiency organic electrophosphorescent devices with tris(2-phenylpyridine)iridium doped into electron-transporting materials

Applied Physics Letters 77, 904 (2000); https://doi.org/10.1063/1.1306639

Organic electroluminescent diodes

Applied Physics Letters 51, 913 (1987); https://doi.org/10.1063/1.98799
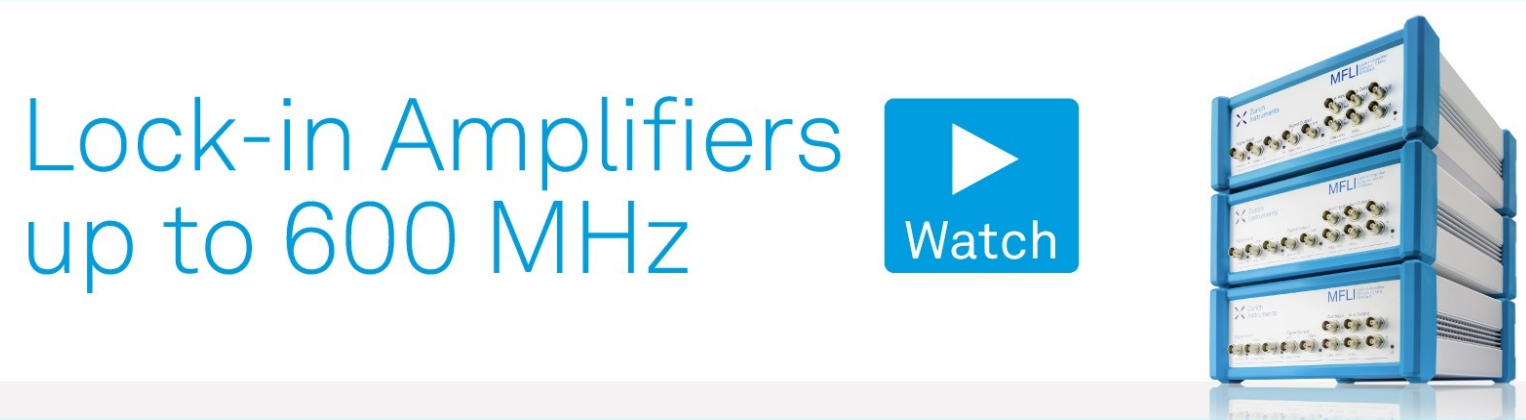


\title{
Electron transport in a starburst oxadiazole
}

\author{
J. Bettenhausen and P. Strohrieg ${ }^{\text {a) }}$ \\ Makromolekulare Chemie I and Bayreuther Institut für Makromolekülforschung (BIMF), \\ Universität Bayreuth, D-95440 Bayreuth, Germany \\ W. Brütting \\ Experimentalphysik II and Bayreuther Institut für Makromolekülforschung (BIMF), Universität Bayreuth, \\ D-95440 Bayreuth, Germany \\ H. Tokuhisa and T. Tsutsui \\ Department of Materials Science and Technology, Graduate School of Engineering Sciences, \\ Kyushu University, Kasuga-shi, Fukuoka 816, Japan
}

(Received 17 April 1997; accepted for publication 6 August 1997)

\begin{abstract}
In this article we report on the electron mobility of a starburst oxadiazole, 1,3,5 -[(4-tert.-butylphenyl)-2-oxadiazolyl] benzene. For direct evidence of the electron transport capability the charge drift mobility and its field dependence have been measured by the time-of-flight technique. The electron mobility showed a square root dependence of the electric field and was somewhat larger than that for PBD. In addition, the oxadiazole compound has been used as the electron transport layer (ETL) in light-emitting diodes (LEDs) together with poly(1,4-phenylene vinylene). The characteristics of these devices are compared to analogous LEDs with $\mathrm{Alq}_{3}$ as the ETL. (C) 1997 American Institute of Physics. [S0021-8979(97)03222-2]
\end{abstract}

\section{INTRODUCTION}

Organic charge transport materials have found a number of technical applications. For many years they have been used as photoconductors in xerography and laser printing. Recently, additional interest came from the rapidly emerging field of organic electroluminescence where charge transport materials have been frequently applied. Depending on the species formed-a radical cation or anion-these compounds are classified as hole or electron transport materials.

One interesting class of such materials is oxadiazoles. It has been known for a long time that derivatives of 1,3,4oxadiazole are photoconductors, e.g. 2,5-(4-diethylaminophenyl)-1,3,4-oxadiazole was frequently used in photocopiers. ${ }^{1}$ There the compound acts as a hole transport material since the electron withdrawing effect of the oxadiazole unit with three electronegative heteroatoms is overcompensated by the two electron donating amino groups. On the other hand, various low molecular weight oxadiazoles like 2-(biphenylyl)-5-(4-tert.-butylphenyl)-1,3,4-oxadiazole (PBD) have been successfully applied in organic multilayer electroluminescence (EL) devices. ${ }^{2,3}$ In this case, the electron withdrawing heterocyclic group dominates the electronic properties and PBD acts as an electron injection and transport layer.

Thin films of such low molar mass oxadiazoles are usually prepared by vacuum evaporation. Although the films are amorphous, they have a strong tendency to crystallize. ${ }^{3}$ One alternative to low molecular weight compounds is guest-host systems in which the compounds are embedded in a polymer matrix-like polymethylmethacrylate. ${ }^{4}$ But the concentration of active molecules in the polymer host is limited due to crystallization of the guest molecules. This can be overcome by covalent fixation of the oxadiazole groups in main

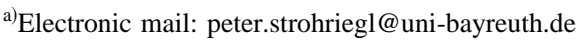

chain ${ }^{5,6}$ or in side group polymers. ${ }^{7,8}$ The main disadvantage of polymers is the lack of efficient purification procedures resulting in ill-defined structures. The latter are in many cases responsible for the bad performance of polymers in electronic applications.

Recently, a new class of materials, star shaped compounds with high glass transition temperatures $\left(T_{g}\right.$ 's), has attracted interest for applications as photoconductors or in organic LEDs. One typical example is $4,4^{\prime}, 4^{\prime \prime}-$ tri(N-carbazolyl)triphenylamine (TCTA). ${ }^{9}$ The molecule forms a stable glass with a $T_{g}$ at $151{ }^{\circ} \mathrm{C}$ and has been successfully applied as a hole transport layer in LEDs. For such molecules, the name starburst is commonly used. ${ }^{9}$

In two preceding papers we have described the synthesis and characterization of a number of starburst molecules and dendrimers with 1,3,4-oxadiazole units. ${ }^{10,11}$ These low molecular weight compounds form stable glasses with glass transition temperatures up to $220^{\circ} \mathrm{C}$.

In this article we report on the electron mobility of the starburst oxadiazole (OXD) (refer to Fig. 1) measured by the time-of-flight (TOF) technique. The novel oxadiazole compound has been used as electron transport layer in LEDs together with poly(1,4-phenylene vinylene) (PPV). Additionally, the characteristics of these devices are compared to analogous LEDs with $\mathrm{Alq}_{3}$ as the ETL.

\section{EXPERIMENTS AND SAMPLE PREPARATION}

\section{A. Time-of-flight measurements}

In the measurements, we have used two-layer type samples with a vacuum deposited film (thickness $=100 \mathrm{~nm}$ ) of a perylene derivative (PV) as the carrier generation layer and a polycarbonate (PC) film doped with $50 \mathrm{wt} \%$ of the OXD (thickness $=$ about $3 \mu \mathrm{m}$ ). For sample preparation, the carrier generation layer of the perylene pigment has been vacuum deposited on an indium-tin-oxide (ITO)-coated glass 


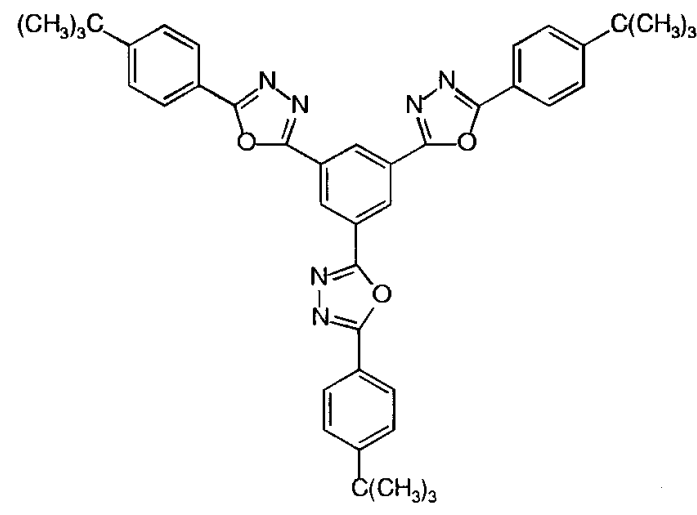

OXD

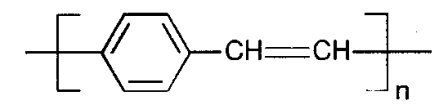

PPV

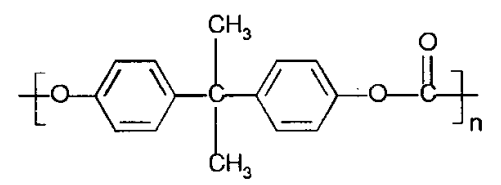

PC

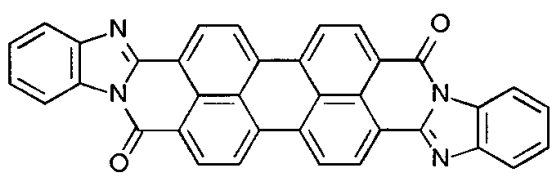

PV

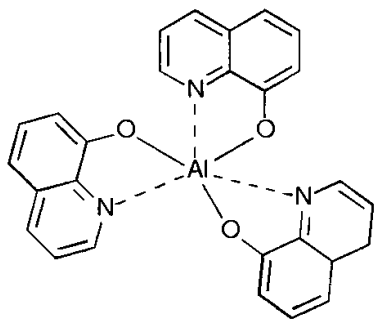

Alq $_{3}$

FIG. 1. Molecular structure of the starburst oxadiazole OXD, poly $(1,4$ phenylene vinylene) (PPV), polycarbonate (PC), the perylene pigment (PV) and $\mathrm{Alq}_{3}$.

substrate first. Then, the doped polycarbonate film has been spin coated on the carrier generation layer from dichloromethane solution. Last, an aluminium counterelectrode has been vacuum deposited. Crystallization has not been found from differential scanning calorimetry (DSC) measurements and optical microscopic observation, although the concentration of the starburst oxadiazole in polycarbonate was rather high.

A dye laser (wavelength $=504 \mathrm{~nm}$ ) has been used as an excitation light source so that carriers are generated only within the layer of the perylene pigment. The photocurrent signals due to transport of the photogenerated carriers have been stored in a transient memory, and time profiles of the photocurrent have been analyzed by a personal computer.

\section{B. Organic light-emitting diodes}

The devices were fabricated by spin coating the PPV precursor solution on ITO substrates and a subsequent thermal conversion of the precursor to the conjugated PPV at $190{ }^{\circ} \mathrm{C}$ in nitrogen atmosphere. In a second step the electron transport layer was deposited by vacuum sublimation of the organic molecules. Then a MgAg cathode was evaporated on top of the structure. The layer thickness was about $100 \mathrm{~nm}$ for PPV, 10-50 nm for the starburst oxadiazole and about $100 \mathrm{~nm}$ for the metal, respectively. The PPV/Alq ${ }_{3}$ heterolayer devices were fabricated in the same way with layer thickness of $50 \mathrm{~nm}$ for $\mathrm{Alq}_{3}$. Current-voltage characteristics and luminance of the devices were measured immediately after preparation under vacuum.

\section{RESULTS AND DISCUSSION}

\section{A. Time-of-flight measurements}

For direct evidence of the electron transport capability of the starburst oxadiazole, the electron drift mobility has been measured by the conventional TOF technique. The TOF measurements have been carried out according to a previous paper where carrier mobilities up to $10^{-5} \mathrm{~cm}^{2} / \mathrm{V}$ s for electrons and $10^{-7} \mathrm{~cm}^{2} / \mathrm{V} \mathrm{s}$ for holes have been found in a number of low molecular weight oxadiazoles depending on their substitution pattern. ${ }^{12}$

Figure 2 shows the transient photocurrent profile for OXD due to electron transport in the doped polycarbonate film at an applied electric field of $1 \times 10^{6} \mathrm{~V} \mathrm{~cm}^{-1}$ at room temperature. An apparent inflection point indicating the transit time $t_{T}$ when the leading edge of the carrier packet reaches the counterelectrode is observed in the profile. From the inflection point $t_{T}$ was determined and the carrier mobility $\mu_{e}$ was calculated according to the equation $\mu$ $=L^{2} / t_{T} F$, where $L$ is the film thickness and $F$ the applied field.

The electric field dependence of the electron drift mobility of the doped polycarbonate film is shown in Fig. 3. It can be described as $\mu \alpha \exp \left(b F^{1 / 2}\right)$, where $b$ is a constant, that has been observed by many workers. ${ }^{13}$ This square root dependence makes hopping as the transport mechanism highly plausible.

The value of the electron drift mobility of the starburst oxadiazole was $1.2 \times 10^{-6} \mathrm{~cm}^{2} \mathrm{~V}^{-1} \mathrm{~s}^{-1}$ at an applied field of $7 \times 10^{5} \mathrm{~V} \mathrm{~cm}^{-1}$ at $273 \mathrm{~K}$. The value is little larger than that of 2-(4-biphenyl)-5-(4-tert.-butylphenyl)-1,3,4-oxadiazole (PBD) doped polycarbonate films, $\mu=8.0$ $\times 10^{-7} \mathrm{~cm}^{2} \mathrm{~V}^{-1} \mathrm{~s}^{-1}$ at the same concentration, electric field 


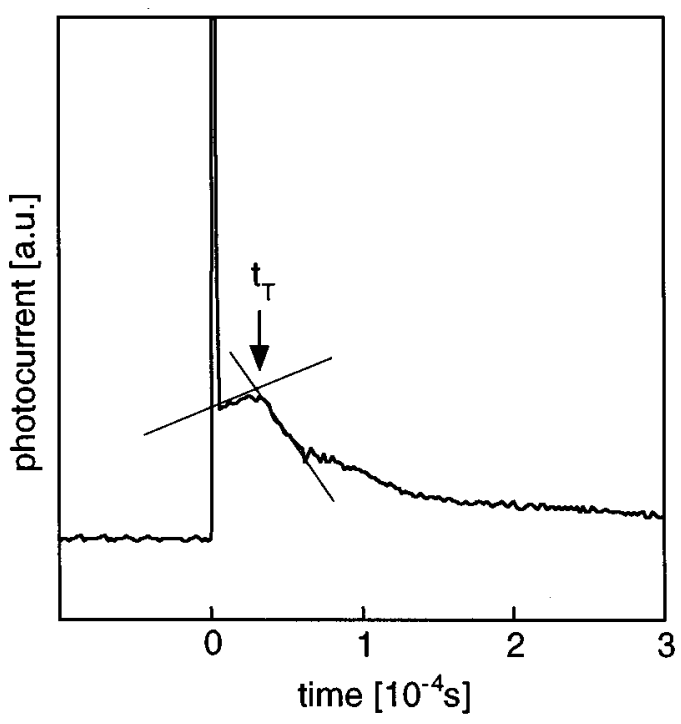

FIG. 2. Transient photocurrent profile due to electron transport in the polycarbonate film doped with $50 \mathrm{wt} \%$ of the starburst oxadiazole at an applied field of $1 \times 10^{6} \mathrm{~V} / \mathrm{cm}^{-1}$ at room temperature.

and temperature, ${ }^{12}$ although OXD has the same chargetransporting chromophore as PBD, tert.-butylphenyloxadiazole, in its molecular structure. From these results, it was confirmed directly that the starburst oxadiazole has good electron-transport capability.

\section{B. Organic light-emitting diodes}

The electron transport capability of the starburst oxadiazole has been used in heterolayer LEDs with PPV as the active emitting material. PPV single layer devices in the configuration ITO/PPV/metal usually show rather poor electroluminescence quantum efficiencies (typically $0.001 \%$ external quantum efficiency for $\mathrm{Al}$ ), since they are majority carrier devices (Schottky diodes) with a large excess hole current leading to an unbalanced current flow. ${ }^{14}$ In previous work we have used oxadiazole polymers spin cast from solution as the electron transport material in heterolayer LEDs and found a significant increase in efficiency of up to two orders of magnitude. ${ }^{5,8}$ Here we make use of the fact that the

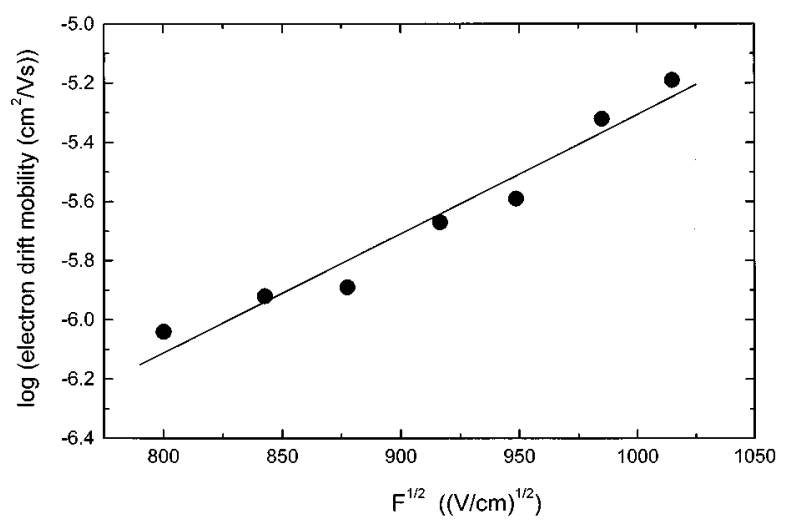

FIG. 3. Electric field dependence of the electron drift mobility of a polycarbonate film doped with $50 \mathrm{wt} \%$ of OXD at $273 \mathrm{~K}$.
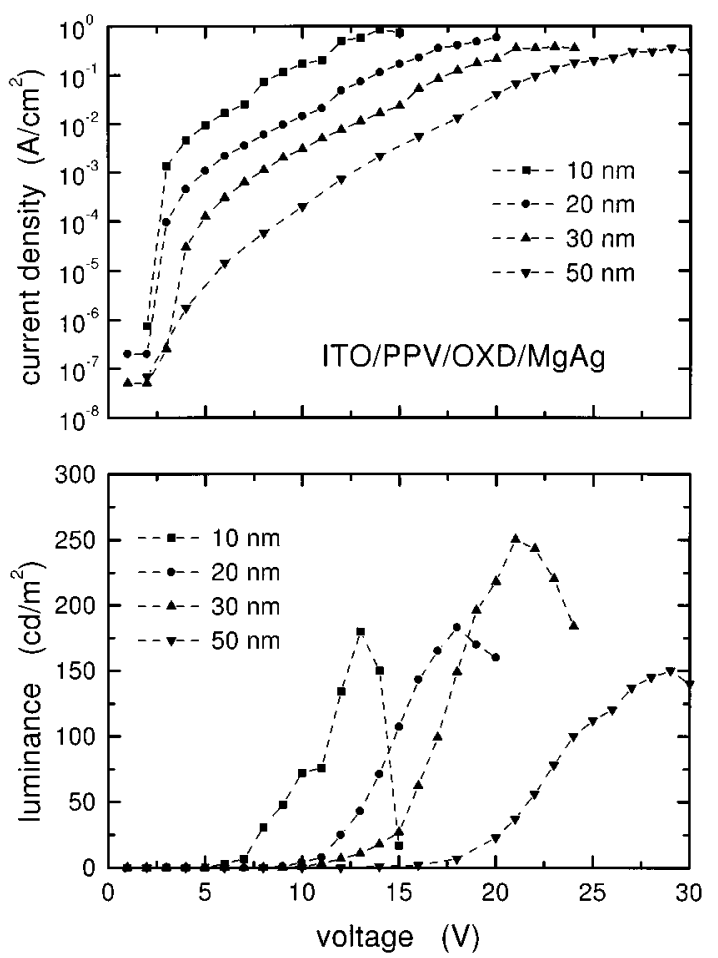

FIG. 4. Current-voltage characteristics (top) and luminance vs voltage (bottom) for different thicknesses of the oxadiazole layer in PPV/oxadiazole devices.

starburst oxadiazole (OXD) is volatile and thin films can be made by vacuum evaporation. For comparison, we have also fabricated heterolayer devices with the well-known electron transport material $\mathrm{Alq}_{3}$.

Figure 4 shows a comparison of $I-V$ curves (top) and luminance versus voltage (bottom) for PPV/oxadiazole devices with different thicknesses of the oxadiazole layer. It can be seen that with increasing oxadiazole layer thickness the current through the device is significantly reduced and at the same time the onset voltage for visible electroluminescence $\left(1 \mathrm{~cd} / \mathrm{m}^{2}\right)$ increases. The maximum brightness in these devices was $300 \mathrm{~cd} / \mathrm{m}^{2}$, which is about a factor of 10 higher than in our best PPV single layer devices. Above a critical voltage the luminance decreases with increasing voltage and the $I-V$ curves flatten, indicating degradation of the device by the high electric field and Joule heating. From the data of Fig. 4 one can calculate the power efficiency of the devices with different oxadiazole layer thickness. The highest values of $0.025 \mathrm{~lm} / \mathrm{W}$ (corresponding to an external quantum efficiency of about $0.1 \%$ ) are obtained for an oxadiazole layer thickness of $30 \mathrm{~nm}$. The other devices show maximum values between 0.010 and $0.015 \mathrm{~lm} / \mathrm{W}$.

The energy level diagrams for the two-layer LEDs are shown in Fig. 5. For PPV, an ionisation potential of $5.0 \mathrm{eV}$ (yielding, together with the band gap of $2.5 \mathrm{eV}$, an electron affinity of $2.5 \mathrm{eV}$ ) has been reported. ${ }^{15}$ From cyclovoltammetric measurements we estimate the highest occupied molecular orbital (HOMO) and lowest occupied molecular orbital (LUMO) energies of OXD as $6.5 \mathrm{eV}$ and $3.2 \mathrm{eV} .^{16}$

One can immediately see from Fig. 5 that there is a large energy barrier for holes from PPV to the oxadiazole. Thus 


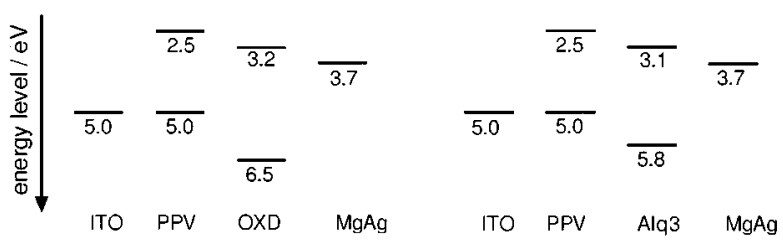

FIG. 5. Energy level diagrams of two-layer LEDs with the starburst oxadiazole (left) and $\mathrm{Alq}_{3}$ (right).

one major effect of the oxadiazole layer is to reduce the large excess hole current in PPV LEDs and therefore improve the charge carrier balance and quantum efficiency. This barrier explains the strong dependence of the device current on the oxadiazole layer thickness seen in Fig. 4. On the other hand, the barrier also leads to hole accumulation at the PPV/ oxadiazole interface, which enhances electron injection.

The strong thickness dependence of the EL onset voltage indicates that the injection of electrons occurs by a field dependent tunneling process. Due to the low conductivity of the oxadiazole it is reasonable to assume that the applied voltage drops almost completely at the oxadiazole layer, which in turn leads to high enough fields for electron tunneling. Additionally, the higher LUMO level of the oxadiazole as compared to PPV significantly reduces the barrier for electron injection. Due to the electron transport capabilities of the oxadiazole the exciton formation and recombination take place in the PPV layer and the emission spectra are (apart from thickness effects) basically the same as for PPV single layer LEDs. Thus a further effect of the oxadiazole layer is to bring the recombination zone away from the metal-polymer interface, which is expected to have a much higher concentration of non-radiative recombination centres.

For comparison, we have also used 8-hydroxyquinolinealuminium $\mathrm{Alq}_{3}$ as the electron transport material on PPV. In this case both PPV and $\mathrm{Alq}_{3}$ can act as emitting materials. However, the comparison of the spectra of PPV/oxadiazole and PPV/Alq ${ }_{3}$ in Fig. 6 clearly shows that the emission in $\mathrm{PPV} / \mathrm{Alq}_{3}$ devices essentially comes from $\mathrm{Alq}_{3}$, since the

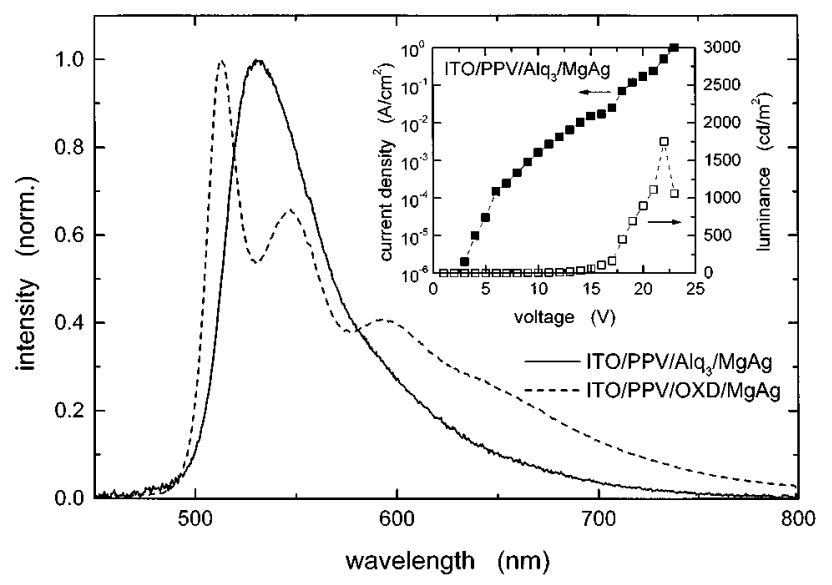

FIG. 6. Electroluminescence spectra of $\mathrm{PPV} / \mathrm{Alq}_{3}$ and PPV/oxadiazole LEDs; the inset shows current-voltage characteristics and luminance vs voltage for $\mathrm{PPV} / \mathrm{Alq}_{3}$ devices. vibronic structure characteristic for PPV is not seen. Additionally, as can be seen from the inset in Fig. 6, these devices do not show saturation in the current at high voltage; they have much higher brightness of about $1500 \mathrm{~cd} / \mathrm{m}^{2}$ and an external power efficiency of more than $0.1 \mathrm{~lm} / \mathrm{W}$ (equivalent to $0.25 \%$ external quantum efficiency).

One important difference in the starburst oxadiazole is the fact that the energy gap of $\mathrm{Alq}_{3}\left(E_{g}=2.7 \mathrm{eV}\right)$ is comparable to PPV. ${ }^{17}$ Thus the offset of the HOMO levels between $\mathrm{PPV}$ and $\mathrm{Alq}_{3}$ is much smaller as compared to PPV/ oxadiazole (see Fig. 5). This leads to a much smaller hole blocking barrier at the PPV/Alq ${ }_{3}$ interface, resulting in a higher total current in these devices.

Regarding the electron injection and transport capability of $\mathrm{Alq}_{3}$, the literature data are comparable to OXD [electron mobility $\mu_{e}=1.4 \times 10^{-6} \mathrm{~cm}^{2} \mathrm{~V}^{-1} \mathrm{~s}^{-1}$ at $E=4 \times 10^{5} \mathrm{~V} / \mathrm{cm}$ (Ref. 18) and the LUMO level $E_{\mathrm{LUMO}}=3.1 \mathrm{eV}$ (Ref. 17)]. However, as the electroluminescence spectra show, the exciton formation and recombination mainly take place in the $\mathrm{Alq}_{3}$ layer. Thus the PPV layer merely acts as the hole transporting material. Moreover, since brightness and efficiency of PPV/Alq ${ }_{3}$ devices are by a factor of 5 higher than in the corresponding PPV/oxadiazole devices, one has to conclude that PPV shows less efficiency for radiative recombination processes than $\mathrm{Alq}_{3}$.

These results are supported by photoluminescence efficiency measurements of PPV films converted onto ITO and quartz substrates. ${ }^{19}$ While our PPV films on glass substrates show photoluminescence quantum efficiencies between $15 \%$ and $20 \%$, it was found that films converted onto ITO substrates show a reduction of photoluminescence by about one order of magnitude. The photoluminescence quenching is caused by $\mathrm{InCl}_{3}$, which is formed during the thermal conversion of the PPV precursor in the reaction of the $\mathrm{HCl}$ leaving group with the ITO substrate. This compound also leads to a doping of PPV, which is responsible for the good hole conducting properties of PPV films on ITO. However, it is not favourable for efficient light emission from PPV. ${ }^{20}$

\section{CONCLUSIONS}

From our results we can conclude that the limiting factor for PPV/oxadiazole heterolayer devices is the rather poor radiative efficiency of PPV converted onto ITO substrates. Thus for light-emitting devices based on precursor PPV, we propose to use chemically stable anode materials or precursor polymers with inert leaving groups. Another possibility is the introduction of an emission layer with high radiative efficiency between PPV and the oxadiazole.

${ }^{1}$ W. Wiedemann, Chem. Ztg. 106, 275 (1982).

${ }^{2}$ C. Adachi, T. Tsutsui, and S. Saito, Appl. Phys. Lett. 55, 1489 (1989).

${ }^{3}$ Y. Hamada, C. Adachi, T. Tsutsui, and S. Saito, Jpn. J. Appl. Phys., Part 131,1812 (1992).

${ }^{4}$ A. R. Brown, D. D. C. Bradley, J. H. Burroughes, R. H. Friend, N. C. Greenham, P. L. Burn, A. B. Holmes, and A. Kraft, Appl. Phys. Lett. 61, 2793 (1992)

${ }^{5}$ E. Buchwald, M. Meier, S. Karg, W. Riess, M. Schwoerer, P. Pösch, H.-W. Schmidt, and P. Strohriegl, Adv. Mater. 7, 839 (1995).

${ }^{6}$ M. Strukelj, F. Papadimitrakopoulus, T. M. Miller, and L. J. Rothberg, Science 267, 1969 (1995).

${ }^{7}$ X. C. Li, F. Cacialli, M. Giles, J. Grüner, R. H. Friend, A. B. Holmes, S. 
C. Moratti, and T. M. Yong, Adv. Mater. 7, 898 (1995).

${ }^{8}$ M. Greczmiel, P. Pösch, H.-W. Schmidt, P. Strohriegl, E. Buchwald, M. Meier, W. Riess, and M. Schwoerer, Macromol. Symp. 102, 371 (1996).

${ }^{9}$ Y. Kuwabara, H. Ogawa, H. Inoda, N. Noma, and Y. Shirota, Adv. Mater. 6, 677 (1994).

${ }^{10}$ J. Bettenhausen and P. Strohriegl, Adv. Mater. 8, 507 (1996).

${ }^{11}$ J. Bettenhausen and P. Strohriegl, Macromol. Rapid Commun. 17, 623 (1996).

${ }^{12}$ H. Tokuhisa, M. Era, T. Tsutsui, and S. Saito, Appl. Phys. Lett. 66, 3433 (1995).

${ }^{13}$ J. Frenkel, Phys. Rev. 54, 647 (1938).

${ }^{14}$ S. Karg, M. Meier, and W. Riess, J. Appl. Phys. 82, 1951 (1997).
${ }^{15}$ D. D. C. Bradley, Synth. Met. 54, 401 (1993).

${ }^{16}$ J. Uebe, J. Salbeck, J. Bettenhausen, and P. Strohriegl (unpublished results).

${ }^{17}$ Y. Shirota, Y. Kuwabara, H. Inada, T. Wakimoto, H. Nakada, Y. Yonemoto, S. Kawami, and K. Imai, Appl. Phys. Lett. 65, 807 (1994).

${ }^{18}$ R. G. Kepler, P. M. Beeson, S. J. Jakobs, R. A. Anderson, M. B. Sinclair, V. S. Valerncia, and P. A. Cahill, Appl. Phys. Lett. 66, 3618 (1995).

${ }^{19}$ M. Herold, C. Drummer, J. Gmeiner, and M. Schwoerer, J. Mater. Sci. (in press).

${ }^{20}$ W. Brütting, M. Herold, M. Meier, S. Karg, and M. Schwoerer, Chem. Phys. (in press). 